\title{
Design considerations of conventional angle diversity receivers for indoor optical wireless communications
}

\author{
Silvestre Rodríguez Pérez ${ }^{1 *}$, Beatriz Rodríguez Mendoza ${ }^{1}$, Rafael Pérez Jiménez , Oswaldo González Hernández \\ and Alberto García-Viera Fernández ${ }^{1}$
}

\begin{abstract}
A conventional angle diversity receiver uses multiple receiving elements that are oriented in different directions, where each element employs its own filter and nonimaging concentrator, such as a compound parabolic concentrator (CPC) or hemispheric lens. In this paper, a study of the design of a conventional receiver structure using angle diversity that offers improved performance with respect to the infrared channel characteristics is presented. To this end, a recently proposed model for the effective signal collection area of a conventional angle diversity receiver that more closely approximates real behaviour than the ideal model is used. The inclusion of this model in a Monte Carlo ray-tracing algorithm allows us to investigate the effects of conventional receiver parameters on the main infrared channel parameters, such as path loss and rms delay spread. Furthermore, in order to determine the number of receiver elements, the outage probability and the average error probability are also considered. Based on the results, a conventional angle diversity receiver composed of seven elements is proposed, with one of them oriented towards the ceiling, and six angled at a $56^{\circ}$ elevation with a $60^{\circ}$ separation in azimuth. For each element, a CPC with a $50^{\circ}$ field of view must be used.
\end{abstract}

Keywords: Angle diversity; Receiver design; Infrared channel; Effective area model; Outage probability

\section{Introduction}

Nondirected infrared (IR) radiation has been considered as a very attractive alternative to radio frequency waves for indoor wireless local area networks. However, there are two major limitations for establishing a wideband infrared communications link: the power requirements and the intersymbol interference caused by multipath dispersion. In general, the use of multibeam transmitter in conjunction with angle diversity receivers makes it possible to reduce the impact of ambient light noise, path loss and multipath distortion, in part by exploiting the fact that they are often received from different directions than the desired signal [1-9]. Basically, the angle diversity detection can be obtained using conventional, imaging or sectored receivers. A conventional receiver uses multiple photodetectors that are oriented in various

\footnotetext{
* Correspondence: srdguezp@ull.es

'Departamento de Física Fundamental y Experimental, Electrónica y Sistemas, Universidad de La Laguna, La Laguna, Tenerife 38203, Spain Full list of author information is available at the end of the article
}

directions [4,5]; an imaging diversity receiver is composed of an optical concentrator that focuses on a segmented photodetector array $[2,6,7]$ and a sectored receiver which is a hemisphere, where a set of parallels and meridians defines the photodetector boundaries $[8,9]$.

The propagation characteristics of the indoor infrared channel are fully described by the channel's impulse response, which depends on multiple factors such as the room geometry, the reflection pattern of surfaces, the emitter and receiver characteristics, and their relative locations. In this paper, we study by simulation those indoor IR links that are characterised by the use of conventional angle diversity receivers. As discussed above, a conventional angle diversity receiver consists of multiple photodetectors that are oriented in various directions, where each receiving element usually employs a band-pass filter and nonimaging concentrator, such as a compound parabolic concentrator (CPC) or hemispheric lens. In order to estimate the impulse response in IR wireless indoor channels, several simulation methods have been put forth

\section{空}


$[10,11]$, but all of them share the same problem, namely, the intensive computational effort. However, we make use of a Monte Carlo ray-tracing algorithm [12,13], which presents a lower computational cost than previous methods, especially when a high temporal resolution and a large number of reflections are required. Indoor optical channel simulation can significantly enhance the design of angle diversity receivers but requires models that correctly fit the receiver characteristics and the remaining elements of the IR channel. That is why, in this work, we use models for the reflection pattern of surfaces, background lightinduced shot noise and an effective signal collection area for a conventional angle diversity receiver that more closely approximate real behaviour than those previously reported. The inclusion of these models in the Monte Carlo ray-tracing algorithm allows us to study more precisely those optical links that are characterised by the use of conventional angle diversity receivers.

This paper is organised as follows. In Section 2, the channel model of the IR link for the conventional receivers using angle diversity and the expression for the calculation of signal-to-noise ratio are defined. Section 3 presents the study for the design of the conventional receiver structure using angle diversity that yields improved performance with respect to the main indoor IR channel parameters. Furthermore, the outage probability and the average error probability are used as a metric for determining the number of receiver elements. Finally, Section 4 outlines the conclusions of this paper.

\section{Channel model and signal-to-noise ratio}

In optical wireless links, the most viable method is to employ intensity modulation (IM), in which the instantaneous power of the optical carrier is modulated by the signal. The receiver makes use of direct detection (DD), where a photodetector generates a current, which is proportional to the instantaneous received optical power. The channel characteristics in an indoor optical wireless channel using IM/DD can be fully characterised by the impulse response $h(t)$ of the channel [1]:

$$
I(t)=R x(t) \otimes h(t)+n(t)
$$

where $I(t)$ represents the received instantaneous current at the output of the photodetector, $t$ is the time, $x(t)$ is the transmitted instantaneous optical power, $\otimes$ denotes convolution, $R$ is the photodetector responsivity and $n(t)$ is the background noise, which is modelled as white and Gaussian, and independent of the received signal.

\subsection{Channel impulse response}

To evaluate the impulse response of the indoor IR channel, a Monte Carlo ray-tracing algorithm was implemented. In general, the impulse response of the IR channel for arbitrary emitter $E$ and receiver $R$ positions can be expressed as an infinite sum of the form [10]

$$
h(t ; E, R)=h^{(0)}(t ; E, R)+\sum_{k=1}^{\infty} h^{(k)}(t ; E, R)
$$

where $h^{(0)}(t ; E, R)$ represents the line-of-sight (LOS) impulse response, and $h^{(k)}(t ; E, R)$ is the impulse response of the light undergoing $k$ reflections, i.e. the multiplebounce impulse responses. Given an emitter $E$ and receiver $R$ in an environment free of reflectors, with a large distance $d$ between both, the LOS impulse response is approximately

$$
h^{(0)}(t ; E, R)=\frac{1}{d^{2}} R_{E}(\phi, n) A_{\mathrm{eff}}(\psi) \delta\left(t-\frac{d}{c}\right)
$$

where $R_{E}(\phi, n)$ represents the generalised Lambertian model used to approximate the radiation pattern of the emitter, $c$ the speed of light and $A_{\text {eff }}(\psi)$ the effective signal collection area of the receiver [10]. In an environment with reflectors, however, the radiation from the emitter can reach the receiver after any number of reflections (see Figure 1). In the algorithm, to calculate the impulse response due to multiple reflections, many rays are generated at the emitter position with a probability distribution equal to its radiation pattern. The power of each generated ray is initially $P_{\mathrm{E}} / N$, where $N$ is the number of rays used to discretize the optical source. When a ray impinges on a surface, the reflection point becomes a new optical source; thus, a new ray is generated with a probability distribution provided by the reflection pattern of that surface. The process continues throughout the maximum simulation time, $t_{\max }$. After each reflection, the power of the ray is reduced by the reflection coefficient of the surface, and the reflected power reaching the receiver $\left(p_{\mathrm{i}, \mathrm{k}}, i\right.$ th ray, $k$ th time interval $)$ is computed by

$$
p_{i, k}=\frac{1}{d^{2}} R_{s}\left(\phi, \phi^{\prime}\right) A_{e f f}(\psi)
$$

where $R_{\mathrm{S}}\left(\phi, \phi^{\prime}\right)$ is the model used to describe the reflection pattern. In this work, Phong's model has been used [13]. In this model, the surface characteristics are defined by three parameters: the reflection coefficient $\rho$, the percentage of incident signal that is reflected diffusely $r_{\mathrm{d}}$ and the directivity of the specular component of the reflection $m$.

Therefore, the total received power in the $k$ th time interval (width $\Delta t$ ) is calculated as the sum of the power of the $N_{\mathrm{k}}$ rays that contribute in that interval. 


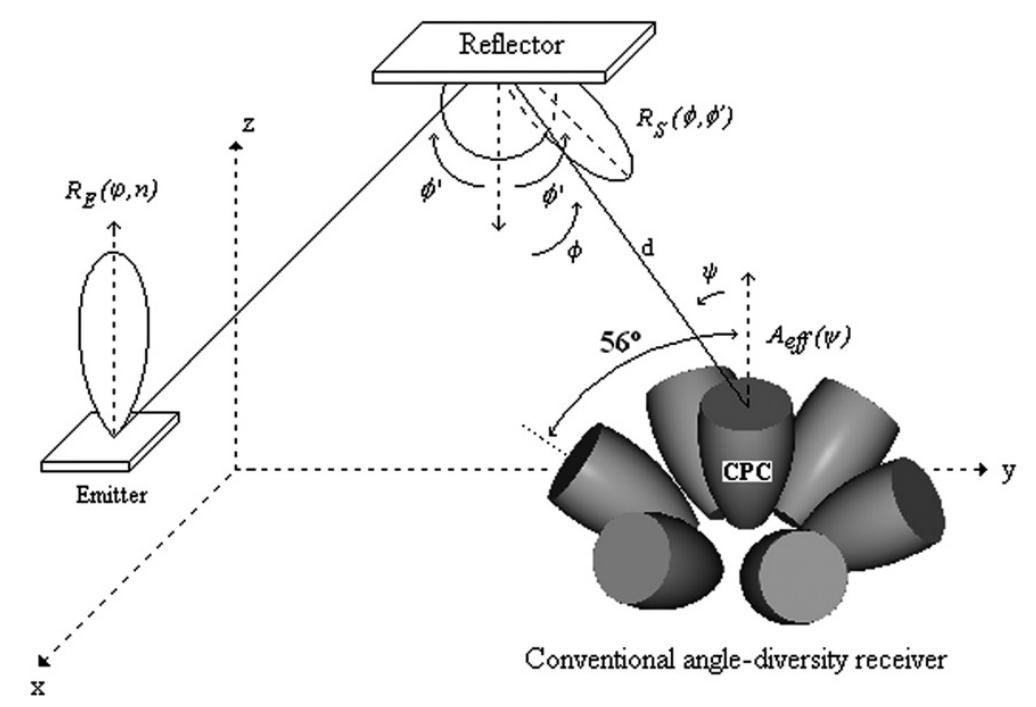

Figure 1 Emitter and receiver geometry with reflector.

$$
p_{k}=\sum_{i=1}^{N_{k}} p_{i, k}=\sum_{i=1}^{N_{k}} \frac{1}{d^{2}} R_{S}\left(\Phi, \Phi^{\prime}\right) A_{\mathrm{eff}}(\psi)
$$

Defining $M=t_{\max } / \Delta t$ and assuming as the time origin the arrival of the LOS component, the impulse response after multiple reflections is given by

$$
\sum_{k=1}^{\infty} h^{(k)}(t ; E, R)=\sum_{j=0}^{M-1} p_{k} \delta(t-j \Delta t) .
$$

Replacing (3) and (6) in (2), the channel impulse response can be expressed as

$$
\begin{aligned}
h(t ; E, R)= & \frac{1}{d^{2}} R_{E}(\phi, n) A_{\mathrm{eff}}(\psi) \delta(t) \\
& +\sum_{j=1}^{M-1} p_{k} \delta(t-j \Delta t) .
\end{aligned}
$$

\subsection{Effective signal collection area model}

A conventional angle diversity receiver uses multiple receiving elements or branches that are oriented in different directions, where each element employs its own filter and nonimaging concentrator, such as a CPC or hemispheric lens. A principal advantage of angle diversity reception is that it allows the receiver to achieve high optical gain and a wide field of view (FOV) simultaneously. Moreover, an angle diversity receiver can reduce the impact of ambient light noise, cochannel interference and multipath distortion.

In general, a bare detector achieves an effective signal collection area of

$$
\begin{aligned}
& A_{\mathrm{eff}}^{\mathrm{bare}}(\psi)=A_{R} \cos (\psi) \operatorname{rect}\left(\frac{\psi}{\mathrm{FOV}}\right) \\
& \operatorname{rect}(x)=\left\{\begin{array}{ll}
1, & |x| \leq 1 \\
0, & |x|>1
\end{array},\right.
\end{aligned}
$$

where $A_{R}$ is the physical area of the receiver, and FOV is the receiver field of view (semi-angle from the surface normal). Adding a filter and concentrator, the effective signal collection area of the receiver becomes

$$
A_{\mathrm{eff}}^{\mathrm{c}, \mathrm{f}}(\psi)=A_{R} T_{S}(\psi) \mathrm{g}(\psi) \cos (\psi) \operatorname{rect}\left(\frac{\psi}{\pi / 2}\right),
$$

where $T_{\mathrm{S}}(\psi)$ is the filter transmission, and $g(\psi)$ is the concentrator gain. Nonimaging concentrators exhibit a trade-off between gain and FOV. An idealised nonimaging concentrator [1] which has an internal refractive index $n$ achieves a constant gain expressed as

$$
g(\psi)=\frac{n^{2}}{\sin ^{2} \psi_{c}} \operatorname{rect}\left(\frac{\psi}{\psi_{c}}\right),
$$

where $\psi_{\mathrm{c}}$ is the concentrator FOV that usually is less than or equal to $\pi / 2$. In our model, the concentrator gain is affected by the optical efficiency $\eta(\psi)$, which represents the reflection losses of the concentrator. Furthermore, the propagation delay introduced by the concentrator is considered [14]:

$$
\begin{aligned}
& g(\psi)=\frac{n^{2}}{\sin ^{2} \psi_{c}} \eta(\psi) \operatorname{rect}\left(\frac{\psi}{\pi / 2}\right) \\
& t(\psi) \neq 0 .
\end{aligned}
$$


Replacing $g(\psi)$ in the expression that defines the effective signal collection area of the receiver, it can be expressed as

$$
A_{\mathrm{eff}}^{c, f}(\psi)=\frac{n^{2} A_{R} T_{S}(\psi) \cos (\psi)}{\sin ^{2} \psi_{c}} \eta(\psi) \operatorname{rect}\left(\frac{\psi}{\pi / 2}\right) .
$$

In addition, in a wireless infrared communications system, an optical band-pass filter can be used to limit the ambient radiation reaching the detector. A common form of band-pass filter consists of a stack of dielectric thin film layers. By properly choosing the number of layers, their thicknesses and their refractive indexes, it is possible to control the surface reflectance and thus the filter transmittance. The filter transmission $T_{\mathrm{S}}(\psi)$ can be described fairly accurately by a simple, five-parameter model [15]. In this model, for radiation of wavelength $\lambda_{\text {o }}$ incident at angle $\psi$, the filter transmission is given by

$$
T\left(\psi ; \Delta \lambda, \psi^{\prime}\right)=\frac{T_{o}}{1+\left[\frac{\lambda_{0}-\lambda^{\prime}\left(\psi ; \psi^{\prime}\right)}{\Delta \lambda / 2}\right]^{2 m}},
$$

where $\psi^{\prime}$ is the filter orientation, $T_{0}$ is the peak transmission at $\psi^{\prime}, \Delta \lambda$ is the spectral half-power bandwidth, $m$ is the filter order and $\lambda^{\prime}\left(\psi ; \psi^{\prime}\right)$ represents the shifting to shorter wavelengths at non-normal incidences, which is described by

$$
\lambda^{\prime}\left(\psi ; \psi^{\prime}\right)=\lambda_{0}\left(\frac{n_{S}^{2}-n_{1}^{2} \sin ^{2} \psi}{n_{S}^{2}-n_{1}^{2} \sin ^{2} \psi^{\prime}}\right)^{1 / 2}
$$

where $n_{1}$ is the index of the input layer and $n_{\mathrm{s}}$ is an effective index for the spacer layer. The design of the optical filter thus boils down to specifying the two parameters $\Delta \lambda$ and $\psi^{\prime}$. The remaining three parameters $\left(n_{\mathrm{s}}, m\right.$ and $\left.T_{0}\right)$ are generally fixed by technology. However, $\Delta \lambda$ is established by the concentrator FOV because the best utilisation of the CPC and filter is provided when its FOV equals [15]. By definition, $T\left(\psi^{\prime}\right)=T_{0}$. The filter FOV can be measured by the angular bandwidth $\Delta \psi$, defined by $T\left(\psi^{\prime}+\Delta \psi\right)=T_{0} / 2$. An estimate of $\Delta \psi$ can be found from Equation (13), with the following approximation being accurate when $\psi^{\prime} \approx 0$ :

$$
\Delta \psi^{\prime} \approx \sin ^{-1}\left[\frac{n_{s}}{n_{1}}\left(\frac{\Delta \lambda}{\lambda_{0}}\right)^{1 / 2}\right] .
$$

\subsection{Error estimation for angle diversity receivers}

The use of an algorithm based on the Monte Carlo method allows for the error in computing the impulse response to be estimated with just one simulation run, as long as the number of rays is large enough. Different error estimations are obtained for several simulations. Nevertheless, we can be confident that the standard deviation of the estimates decreases as the number of rays is increased. Moreover, the method allows for the accuracy of the results to be assessed. The partial results of one simulation can also be used to achieve a more accurate solution by selecting a suitable number of rays.

In the previous research $[16,17]$, the equation that provides an error determination when computing the impulse response for receivers using a single-element detector was reported and can be estimated as the square root of the total received power variance, $\operatorname{var}\left(p_{\mathrm{k}}\right)$, in the $k$ th time interval (width $\Delta t$ ).

$$
\begin{aligned}
\operatorname{err}\left(p_{k}\right) & =\sqrt{\operatorname{var}\left(p_{k}\right)} \\
& =\sqrt{\sum_{i=1}^{N_{k}} p_{i, k}^{2}-\frac{1}{N}\left(\sum_{i=1}^{N_{k}} p_{i, k}\right)^{2}},
\end{aligned}
$$

where $p_{\mathrm{i}, \mathrm{k}}$ is the reflected power reaching the receiver (ith ray, $k$ th time interval), $N_{\mathrm{k}}$ are the rays that contribute in that interval, $N$ is the number of rays used to discretize the source and $p_{\mathrm{k}}$ is the total received power, which was described in (5). Therefore, the relative error in a time interval $\Delta t$ can be expressed as

$$
\text { rel } \operatorname{err}\left(p_{k}\right)=\sqrt{\frac{\sum_{i=1}^{N_{k}} p_{i, k}^{2}}{\left(\sum_{i=1}^{N_{k}} p_{i, k}\right)^{2}}-\frac{1}{N}} .
$$

These equations can be generalised for an angle diversity receiver composed of $L$ branches with equal-gain combining (EGC). Therefore, the relative error in a time interval $\Delta t$ is given by

$$
\operatorname{rel} \operatorname{err}\left(p_{k}\right)=\sqrt{\frac{\sum_{j=1}^{L} \sum_{i=1}^{N_{k}} p_{i, k}^{2}}{\left(\sum_{j=1}^{L} \sum_{i=1}^{N_{k}} p_{i, k}\right)^{2}}-\frac{1}{N}} .
$$

Figure 2 shows the impulse response and its relative error for an IR link set into room B (see Figure 3), employing a conventional angle diversity receiver with an equal-gain combining, which is composed of seven branches or detectors, one oriented vertically towards the ceiling, and six at a $56^{\circ}$ elevation with a $60^{\circ}$ separation in azimuth. For each detector, a CPC with a FOV or $\psi_{\mathrm{c}}=50^{\circ}$ was used. The number of rays used in the simulation was 100,000 . While the emitter was located in the centre of room, $1 \mathrm{~m}$ above the floor and aimed vertically towards the ceiling, the conventional receiver was located $0.5 \mathrm{~m}$ from the emitter in the $x$ direction: $x=3.5$ and $y=6.57$. The remaining parameters used in the simulation match those shown in Table 1. Figure 2 shows that the relative error estimation is less than 5\%; however, as the Monte Carlo method establishes that the error is proportional to $1 / \sqrt{N}$, the number of rays needed to achieve the results with a given accuracy can 

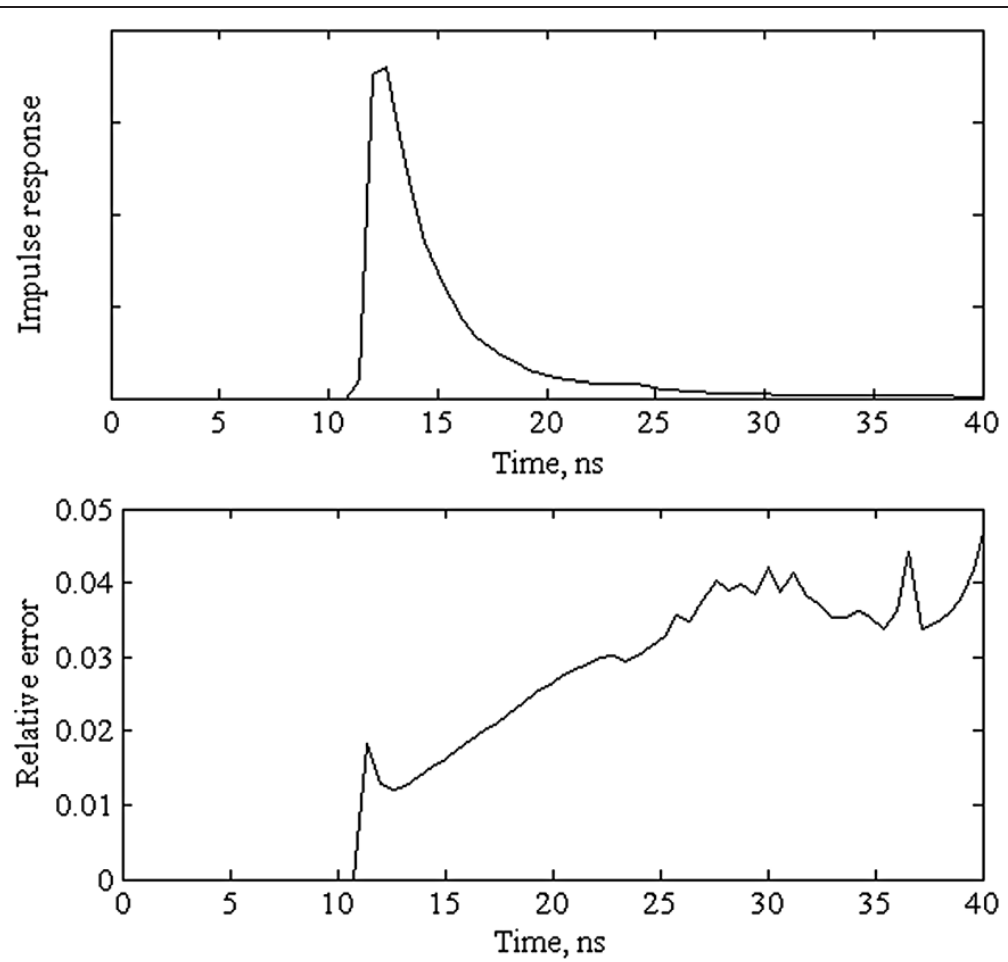

Figure 2 Simulated impulse response and relative error estimation for 100,000 rays.

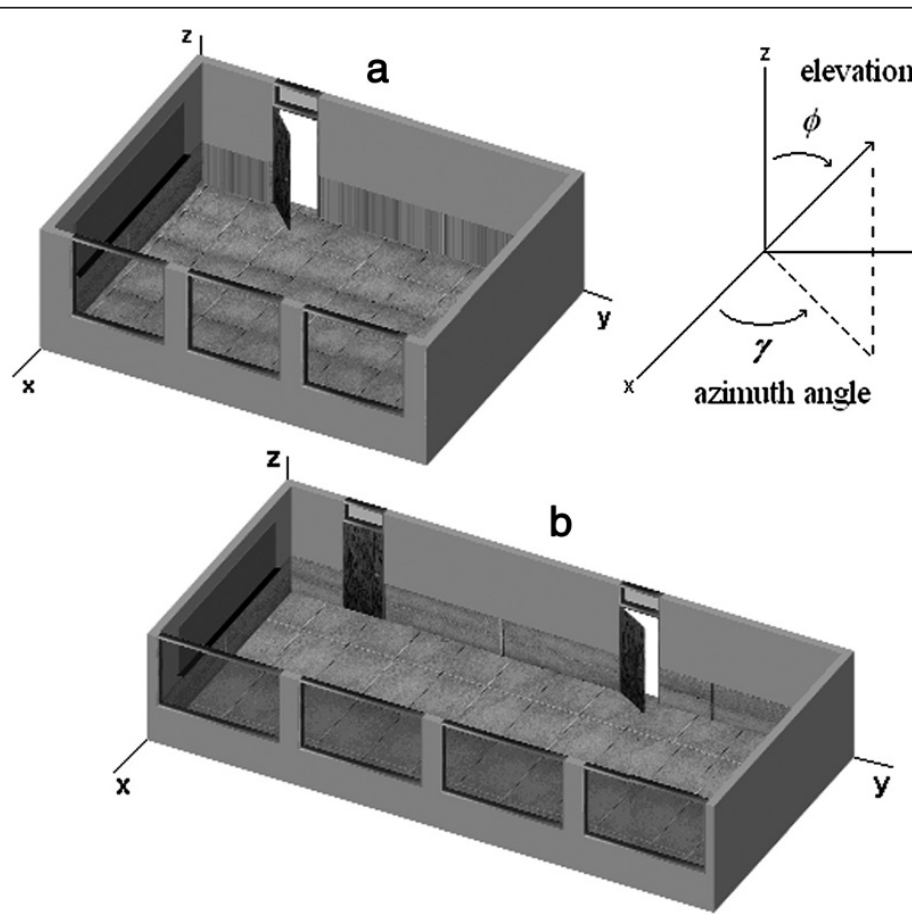

Figure 3 Graphical representation of rooms a and $b$. 
Table 1 Simulation parameters

\begin{tabular}{|c|c|c|c|}
\hline Element & Parameter & A & B \\
\hline \multirow[t]{3}{*}{ Room } & Width $(x), m$ & 6 & 6 \\
\hline & Length $(y), m$ & 7.8 & 13.14 \\
\hline & Height (z), m & 2.75 & 2.75 \\
\hline \multirow[t]{3}{*}{ Emitter } & Mode $(n)$ & 1 & 1 \\
\hline & Power $\left(P_{\mathrm{E}}\right), \mathrm{W}$ & 0.4 & 0.4 \\
\hline & Position $(x, y, z), m$ & $(3,3.9,1)$ & $(3,6.57,1)$ \\
\hline \multirow[t]{4}{*}{ IR detector } & Active area $\left(A_{R}\right), \mathrm{mm}^{2}$ & 2 & 2 \\
\hline & Height (z), m & 1 & 1 \\
\hline & Responsivity (R), A/W & 0.6 & 0.6 \\
\hline & Minimum power detected, $\mathrm{W}$ & $10^{-12}$ & $10^{-12}$ \\
\hline \multirow[t]{2}{*}{ Concentrator } & Refractive index & 1.8 & 1.8 \\
\hline & Exit aperture, mm & 0.8 & 0.8 \\
\hline \multirow[t]{6}{*}{ Band-pass filter } & Number of layers & 20 & 20 \\
\hline & Peak transmission $\left(T_{0}\right)$ & 0.92 & 0.92 \\
\hline & Effective index $\left(n_{5}\right)$ & 2.293 & 2.293 \\
\hline & Filter order $(m)$ & 3 & 3 \\
\hline & Angular bandwidth $(\Delta \psi)$ & $\psi_{c}$ & $\psi_{c}$ \\
\hline & $\lambda_{0}, \mathrm{~nm}$ & 810 & 810 \\
\hline \multirow[t]{2}{*}{ Tungsten lamps } & The corner lamp is located at $(x, y)$ & - & $(1.5,3.57)$ \\
\hline & Lamp spectral density, W/nm & - & 0.037 \\
\hline \multirow[t]{2}{*}{ Windows } & Diffused solar radiation, $\mathrm{W} / \mathrm{m}^{2}$ & 10 & 10 \\
\hline & Window area, $\mathrm{m}^{2}$ & 3.6 & 5.3 \\
\hline \multirow[t]{3}{*}{ Simulation } & Resolution $(\Delta t), n s$ & 0.2 & 0.2 \\
\hline & Bounces (k) & 20 & 20 \\
\hline & Number of rays $(N)$ & 500,000 & 500,000 \\
\hline \multirow[t]{5}{*}{ Materials } & Wood $\left(\rho, r_{\mathrm{d}}, m\right)$ & $(0.63,0.6,3)$ & $(0.63,0.6,3)$ \\
\hline & Varnished wood $\left(\rho, r_{d} m\right)$ & $(0.75,0.3,97)$ & $(0.75,0.3,97)$ \\
\hline & Cement $\left(\rho, r_{d}, m\right)$ & $(0.40,1.0,-)$ & $(0.40,1.0,-)$ \\
\hline & Ceramic floor $\left(\rho, r_{\mathrm{d}}, m\right)$ & $(0.16,0.7,20)$ & $(0.16,0.7,20)$ \\
\hline & Glass $\left(\rho, r_{\mathrm{d},} m\right)$ & $(0.03,0.0,280)$ & $(0.03,0.0,280)$ \\
\hline
\end{tabular}

be easily calculated. For example, if the number of rays used is 500,000, the relative error estimation will be less than $2.24 \%(5 \% \sqrt{100,000} / \sqrt{500,000})$.

\subsection{Signal-to-noise ratio}

In general, the signal-to-noise ratio (SNR) can be expressed by

$$
S N R=\left(R P_{S}\right)^{2} / \sigma^{2}
$$

where $P_{\mathrm{S}}$ is the optical signal power, $R$ is the photodetector responsivity and $\sigma^{2}$ represents the total noise variance, which is the sum of the contributions from the background light-induced shot noise and thermal noise due to the amplifier [18]:

$$
\sigma^{2}=\sigma_{\text {shot }}^{2}+\sigma_{\text {thermal }}^{2}
$$

The shot noise variance can be approximated by

$$
\sigma_{\text {shot }}^{2} \approx 2 q R I R_{b} P_{b g},
$$

where $q=1.6 \times 10^{-19} \mathrm{C}$ is the electron charge, $I=0.562$ is a noise bandwidth factor, $R_{\mathrm{b}}$ is the bit rate and $P_{\mathrm{bg}}$ is the incident optical power from ambient light (natural and artificial). In the rooms considered in this work, the ambient light results from the windows and six tungsten lamps in the ceiling (see Figure 3). To calculate the incident optical power from windows, each window surface is divided into small elements of equal area $\left(0.04 \mathrm{~m}^{2}\right)$, with each element modelled as a first-order Lambertian emitter with a radiant emittance of $10 \mathrm{~W} / \mathrm{m}^{2}$. Applying the Monte Carlo ray-tracing algorithm for each element $E$ and according to Equation (7), the incident optical power from the windows act as an ambient light (noise) source that can be expressed as a sum of the form:

$$
\begin{aligned}
P_{b g} & =\sum_{j=1}^{N_{e}}\left(\sum_{j=0}^{M} h(t ; E, R)\right) \\
& =\sum_{j=1}^{N_{e}}\left(\frac{1}{d^{2}} R_{E}(\varphi, 1) A_{\mathrm{eff}}(\psi)+\sum_{j=1}^{M-1} P_{k}\right),
\end{aligned}
$$

where $M=t_{\max } / \Delta t$ is the number of time intervals of width $\Delta t$, and $N_{\mathrm{e}}$ is the number of elements used to divide the window surface. Moreover, the radiant intensity from the lamps can be modelled as Lambertian sources of second order with optical spectral density of 0.037 $\mathrm{W} / \mathrm{nm}$. The incident optical power from the lamp can be obtained by multiplying the optical spectral density by $\Delta \lambda$, the spectral half-power bandwidth of the optical filter used to limit the ambient radiation reaching the detector. As in the previous case, the optical power contribution from a lamp can be calculated by

$$
P_{b g}=\frac{1}{d^{2}} R_{E}(\varphi, 2) A_{\mathrm{eff}}(\psi)+\sum_{j=1}^{M-1} p_{k} .
$$

Assuming the use of a FET-based transimpedance preamplifier [1], the thermal noise variance can be expressed as

$$
\begin{aligned}
\sigma_{\text {thermal }}^{2}= & \frac{4 k T}{R_{f}} I_{2} R_{b} \\
& +\frac{16 \pi^{2} k T}{g_{m}}\left(\Gamma+\frac{1}{g_{m} R_{D}}\right) C_{T}^{2} I_{3} R_{b}^{3} \\
& +\frac{4 \pi^{2} K I_{D}^{a} C_{T}^{2}}{g_{m}^{2}} I_{f} R_{b}^{2},
\end{aligned}
$$

where $k$ is Boltzmann's constant, $T$ is absolute temperature, $R_{\mathrm{f}}$ is the feedback resistor, $g_{\mathrm{m}}$ is the transconductance, $C_{\mathrm{T}}$ is the total input capacitance of 
receiver, $R_{D}$ is the polarisation resistance, $\Gamma$ is the FET channel noise factor, $K$ and $a$ are the FET $1 / f$ noise coefficients, $I_{\mathrm{D}}$ is the FET drain current, and $I_{2}, I_{3}$ and $I_{\mathrm{f}}$ are noise-bandwidth factors. In this paper, we consider that the thermal noise at the receiver amplifier is negligible compared to the shot noise. In order to perform the comparison, we evaluated the shot noise and thermal noise variances for an IR diffuse link placed in room B (see Figure 3). The remaining parameters used in the simulation match those shown in Table 1 . In room B, the ambient light is provided by four windows and six tungsten lamps in the ceiling. The average shot noise variance obtained is $2.10 \times 10^{-15} \mathrm{~A}^{2}$, which was evaluated at over 400 different locations of a receiver based on a single detector element, oriented vertically toward the ceiling, and with a physical area of $2 \mathrm{~mm}^{2}$. Assuming the parameters that might be typical of a receiver operating in a $100 \mathrm{Mbps}$ diffuse link, i.e. $k=1.38 \times 10^{-23} \mathrm{~J} / \mathrm{K}$, $T=295 \mathrm{~K}, R_{\mathrm{f}}=10 \mathrm{k} \Omega, g_{\mathrm{m}}=40 \mathrm{mS}, C_{\mathrm{T}}=4.5 \mathrm{pF}$ (for 2 $\mathrm{mm}^{2}$ physical area), $R_{\mathrm{D}}=146 \Omega, \Gamma=1.5, K=294 \mathrm{fA}$, $a=1, I_{\mathrm{D}}=20 \mathrm{~mA}, I_{2}=0.562, I_{3}=0.0868$ and $I_{\mathrm{f}}=0.184$, the thermal noise variance obtained is $9.15 \times 10^{-17} \mathrm{~A}^{2}$. As we can observe, the thermal noise is two orders of magnitude lower than the shot noise.

For an angle diversity receiver composed of $L$ branches with EGC, the noise variance EGC is found by summing the total noise variance over all the branches or channels, i.e.

$$
\sigma_{\mathrm{EGC}}^{2}=\sum_{i=1}^{\mathrm{L}} \sigma_{i}^{2}
$$

\section{Study of the design of a conventional angle diversity receiver}

The algorithm described in the previous section, including the conventional receiver model, was implemented.
In what follows, several simulation results obtained for different optical links that are characterised by the use of conventional angle diversity receivers are studied. Using these results, it is possible to establish those parameters of the receiver structure that offer the best performance with respect to the IR channel features.

\subsection{Effects of receiver parameters on the IR channel}

In order to investigate the effects of the design of the parameters of a conventional receiver on the IR channel characteristics, the impulse response $h(t)$, the path loss $(P L)$ and rms delay spread $(D)$ for different configurations of optical links were computed. Once $h(t)$ has been computed, $P L$ and $D$ are easily calculated [1]. To this end, the IR signal propagation in the room B was studied. Figure 3 shows the graphical representation of the room and Table 1, the parameters used in the simulations. In order to ensure that any contribution above the minimum power detected by the photodetector is computed, the maximum number of reflections has been fixed to 20. The emitter is located at the centre of the room, $1 \mathrm{~m}$ above the floor and aimed towards the ceiling, and the IR detector is located at $3 \mathrm{~m}$ from the emitter, in the southwest direction on the diagonal: $x=5.2$ and $y=4.4$.

To evaluate the rotation effects of the IR detector on the received signal, the azimuth angle, $\gamma$, was varied from $0^{\circ}$ to $360^{\circ}$ in steps of $36^{\circ}$ for a specific elevation angle of $\phi=30^{\circ}$ (see Figure 3). Figure 4a,b shows the rms delay spread and the path loss, respectively, as functions of the detector azimuth angle for several FOVs: $20^{\circ}, 30^{\circ}, 40^{\circ}$, $50^{\circ}, 70^{\circ}$ and $90^{\circ}$. For FOVs from $20^{\circ}$ to $70^{\circ}$, a CPC with 1.8 refractive index and $5.64-\mathrm{mm}$ exit aperture were considered, while a FOV $=90^{\circ}$ indicates that an hemispheric lens was employed. Independently of FOV, the losses are minimal for a $135^{\circ}$ azimuth angle since for this
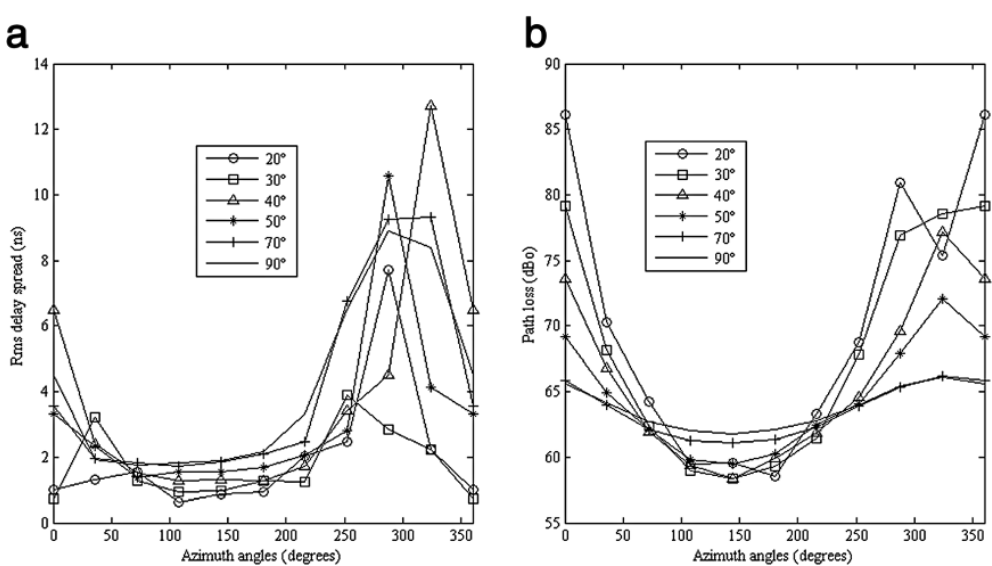

Figure 4 Channel parameters as a function of azimuth angle for a $30^{\circ}$ elevation angle and several FOVs. (a) rms delay spread. (b) Path loss. 
angle, the receiver is facing the centre of the ceiling, where nearly all of the radiation is coming from. Besides, for this orientation, the use of receivers with a reduced FOV provides a more power-efficient link, due mainly to the increased gain offered by the concentrators with a reduced FOV. The greatest losses are obtained with the receiver facing towards the corner of the room and with reduced FOVs, since receivers with a wide FOV are more power efficient since they gather all the radiation coming in from multiple directions. The minimum of the curves that define the delay spread matches that of the propagation losses, $\gamma=135^{\circ}$, since at this orientation, almost all of the power reaching the receiver from the emitter does so after just one reflection. As the receiver is oriented away from the centre of the ceiling in the room, the number of duplicates of the original signal arriving at the receiver with different propagation times escalates, which leads to an increase in the rms delay spread. This increase is proportional to the FOV of the receiver.

Furthermore, the normalised autocorrelation of the rms delay spread has been calculated, which allows us to describe the relation between the channel features seen by the detector and its FOV. The curves corresponding to the normalised autocorrelation show how correlated are the channel rms delay spreads when rotating the receiver by $n$ degrees in azimuth (see Figure 5). For an elevation angle of $30^{\circ}$, a FOV $=40^{\circ}$ and assuming that a new channel is seen by the receiver when the correlation drops below 0.4 [19], we can have independent branches or channels of a conventional angle diversity receiver at angular separations of $66.2^{\circ}$, that is, under said conditions, as the receiver's azimuth angle is rotated, it can distinguish among $360^{\circ} / 66.2^{\circ} \cong 5$ channels with different characteristics. Table 2 shows the number of elements that would be obtained for each of the FOVs studied. In principle, it is expected that as the FOV is increased, the number of

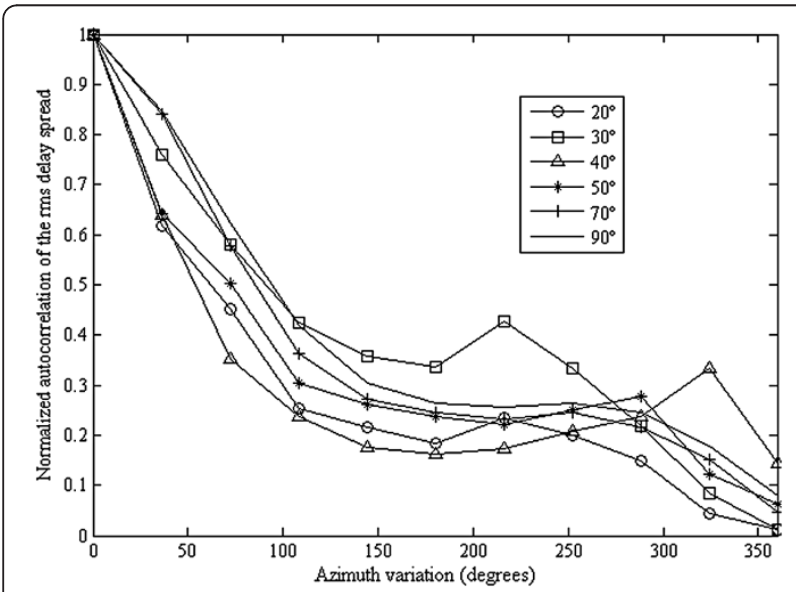

Figure 5 Normalised autocorrelation of the rms delay spread for a $30^{\circ}$ elevation angle and several FOVs.
Table 2 Number of elements obtained as a function of FOV for a $30^{\circ}$ elevation angle

\begin{tabular}{ccc}
\hline FOV (deg) & Azimuth variation (deg) & Number of elements \\
\hline 20 & 81.5 & 4 \\
30 & 121.1 & 3 \\
40 & 66.2 & 5 \\
50 & 90.4 & 4 \\
70 & 101.9 & 3 \\
90 & 114.4 & 3 \\
\hline
\end{tabular}

elements decreases. Though this is usually the case, such an inverse proportionality is not guaranteed. For example, for a $40^{\circ} \mathrm{FOV}$, the number of independent channels is greater than that for $30^{\circ}$. From the resulting data, it can be observed that if a hemispheric lens $\left(\mathrm{FOV}=90^{\circ}\right)$ with three branches separated by $114.4^{\circ}$ is used for the IR detector, three azimuth independent channels are available.

With the same configuration as that used for the azimuth study, the elevation angle was varied from $0^{\circ}$ to $90^{\circ}$ in steps of $10^{\circ}$, and the channel parameters for the same FOVs were calculated. As with the azimuth study, the curves that define the normalised autocorrelation of the rms delay spread for each FOV allow for a determination to be made of the degree of similarity of the channels seen by each detecting element, and its FOV as the elevation angle is varied. Table 3 shows the number of detectors that give rise to independent channels as a function of FOV and for a $180^{\circ}$ azimuth angle. Likewise, it follows that the number of elements in elevation is similar when using either a hemispheric lens or a CPC with a FOV greater than $20^{\circ}$. This result is only valid for the azimuth angle considered. In brief, for a $30^{\circ}$ elevation angle and a $40^{\circ} \mathrm{FOV}$, if elements are oriented every $62.2^{\circ}$ in azimuth, five channels with different characteristics will be available, while for a $180^{\circ}$ azimuth angle and the same FOV, if the elements are separated $42.2^{\circ}$ in elevation, two independent channels will result.

\subsection{Effects of receiver parameters on the IR channel regardless of the receiver location}

The study of the dependence of the IR channel characteristics on the parameters that define the structure of a

\begin{tabular}{|c|c|c|}
\hline FOV (deg) & Elevation variation (deg) & Number of elements \\
\hline 20 & 15.5 & 6 \\
\hline 30 & 37.9 & 2 \\
\hline 40 & 42.2 & 2 \\
\hline 50 & 42.0 & 2 \\
\hline 70 & 40.4 & 2 \\
\hline 90 & 44.0 & 2 \\
\hline
\end{tabular}


conventional angle diversity receiver provided a procedure for selecting the location of the detectors of the receiver so as to yield channels with different characteristics (independent channels). The results of the study detailed in the previous section, however, are only valid for the receiver location considered, namely, the southwest corner of room B. Moreover, the angular arrangement of the elements was obtained following an azimuth study carried out for a specific elevation angle of $30^{\circ}$ and an elevation study for a specific azimuth angle of $180^{\circ}$. If a structure completely independent of receiver location and valid for all elevation and azimuth angles is desired, the analysis must be extended to consider all possible receiver positions and elevation and azimuth angles. So as to gather the necessary data to conduct such a study, multiple simulations were carried out using the two rooms shown in Figure 3. The emitter was located at the centre of each room, $1 \mathrm{~m}$ above the floor and aimed vertically towards the ceiling. The receiver was also located on a plane $1 \mathrm{~m}$ above the floor and was moved in concentric circles with respect to the emitter position. On each circle, spaced $0.5 \mathrm{~m}$ apart, eight uniformly spaced positions were considered. In each location, the impulse response was determined for receivers with FOVs from $10^{\circ}$ to $90^{\circ}$ in steps of $10^{\circ}$ for 100 different orientations. The remaining parameters used in the simulations matched those shown in Table 1. As in the previous section and based on the measurement plan presented, we conducted a study of the dependence of the channel characteristics on the FOV and orientation of a detecting element.

Figure $6 \mathrm{a}, \mathrm{b}$ shows the average and standard deviation, respectively, of the angular separation in elevation that results in channels with independent characteristics as a function of azimuth angle for several FOVs. The procedure used to compile the graphs is similar to that used in the previous section. Starting from the normalised autocorrelation for the rms delay spread in elevation, we determined the angular separation in elevation required for the rms delay spread to fall below 0.4, along with the average and standard deviation of the values obtained for each of the azimuth angles analysed. Independently of the azimuth angle, the angular separation in elevation that provides independent channels for each FOV is determined by the sum of the maximum of the average angular separation for said FOV and its standard deviation. For example, for a $50^{\circ} \mathrm{FOV}$, the resulting angular separation is approximately $56^{\circ}$ in elevation $\left(\cong 47^{\circ}+9^{\circ}\right)$.

The next step involves selecting the FOV of the detecting elements. Doing so requires studying the parameters that characterise the channel as a function of FOV and elevation angle. Figure 7a,b shows, respectively, the average rms delay spread and path loss as a function of elevation angle for several FOVs. These curves were prepared after analysing all the orientations in azimuth for each possible receiver position within the two rooms. The curves allow us to deduce that detectors with small FOVs result in lower rms delay spread and path loss values than those with wide FOVs. As an example, note that using a hemispheric lens, $\mathrm{FOV}=90^{\circ}$, is the best option for those elements with orientations close to $90^{\circ}$ and the worst when dealing with elevations near $0^{\circ}$ (from the vertical). In general, in order to obtain a conventional angle diversity receiver with similar elements, CPCs with a $50^{\circ} \mathrm{FOV}$ should be used, as this offers the best compromise in both parameters regardless of the elevation angle in question.

A similar procedure for the study in elevation was executed in azimuth, i.e. the average and standard deviation of the angular separation in azimuth that result in independent channels as a function of elevation angle for several FOVs were studied. The procedure used to compile both parameters is similar to that used for the study in elevation. Starting from the normalised autocorrelation for
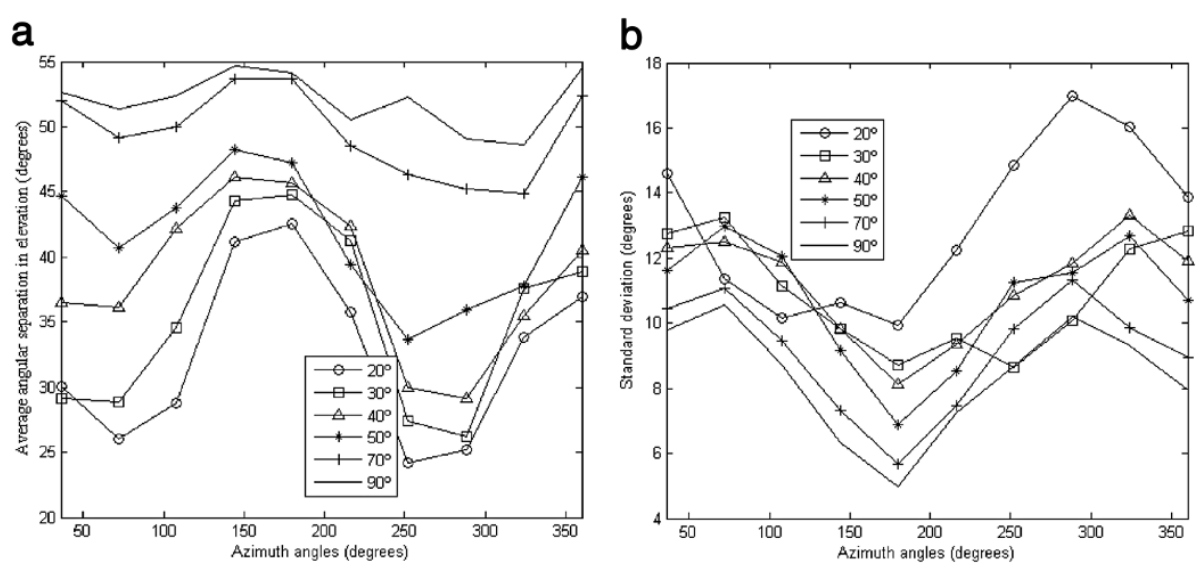

Figure 6 Elevation study as a function of azimuth angle for several FOVs. (a) Average angular separation in elevation that yields independent channels. (b) Standard deviation. 


\section{a}

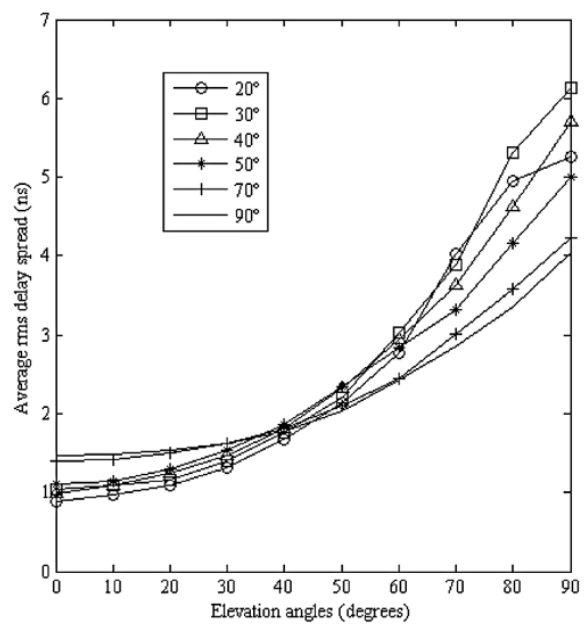

b

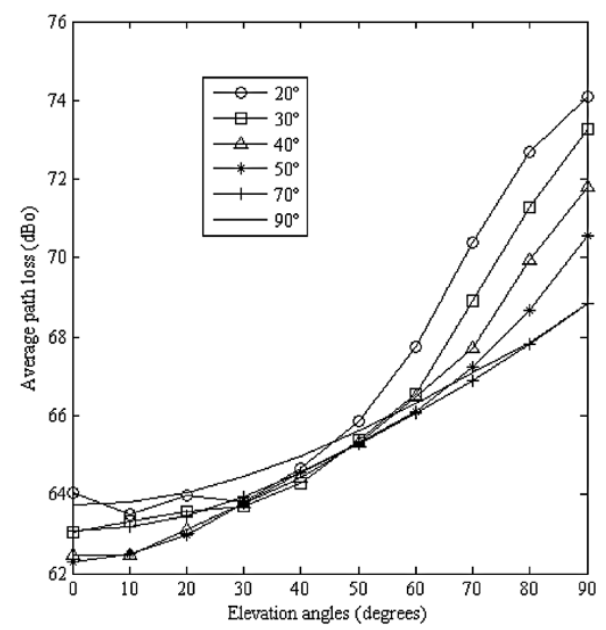

Figure 7 Channel parameters as a function of elevation angle for several FOVs. (a) Average rms delay spread. (b) Average path loss.

the rms delay spread in azimuth, we determined the angular separation in the azimuth required for the rms delay spread to fall below 0.4 , along with the average and standard deviation of the values obtained for each of the elevation angles analysed. According to the obtained results and independently of FOV, the receivers with elevation angles close to zero present angular separations in azimuth greater than those with near horizontal orientations or high elevation angles. This fact implies a minor number of receivers distributed in azimuth for small elevation angles. For a $50^{\circ} \mathrm{FOV}$, the resulting angular separation is approximately $235^{\circ}$ in azimuth, i.e. it is not possible to have two or more receivers to verify this condition $\left(360^{\circ} / 235^{\circ}=\right.$ 1.5). Therefore, the number of elements in azimuth must be localised in order that the receiver has axial symmetry and to cover all possible directions of reception. As in the elevation study, the average rms delay spread and path loss as a function of azimuth angle for several FOVs were computed. Both parameters were obtained after analysing all the orientations in elevation for each possible receiver position within the two rooms. For selected FOV, both parameters present less variation with respect to the azimuth angle and offer the best performance in both parameters, i.e. the lowest values of rms delay spread and path loss, simultaneously.

\subsection{Selecting the number of receiver branches: outage probability and average error probability}

In summary, the results of the elevation and azimuth analysis only revealed that the conventional receiver must consist of detector elements with concentrators with a $50^{\circ} \mathrm{FOV}$, where one is oriented vertically towards the ceiling, and the rest are in a uniform azimuthal arrangement, forming a $56^{\circ}$ angle with respect to the vertically oriented detector. So as to determine the number of elements or branches to be arranged in azimuth, this section presents a study similar to that conducted in [20], where the outage probability and the average error probability are used as measures of link quality to analyse angle diversity at the receiver, the objective being to determine the number of branches required. In this paper, the outage probability is defined by the percentage of receiver locations corresponding to an error probability greater than $10^{-9}$, as reported in [20].

In order to calculate the outage probability and the average error probability, the IR channel model described in Section 2 using 2-pulse-position modulation (2-PPM) for eight arrangements of conventional angle diversity receivers was studied. PPM is an orthogonal modulation scheme that offers a lower average power requirement to achieve a desired bit error rate compared with on-off keying (OOK), at the expense of an increased bandwidth requirement [1]. This decreased average power requirement makes PPM especially suitable for diffuse links (nondirected nonline-of-sight IR links). Furthermore, PPM can achieve much greater immunity than OOK to near-DC noise from fluorescent lamps. For these reasons, PPM is employed in the two main commercial wireless infrared systems for transmitting high data rates: the IrDA and IEEE 802.11 standards. The continuous-time block diagram of an L-PPM system is shown in Figure 8a [21]. Each group of $\log _{2} L$ bits is encoded into an L-PPM waveform $p_{\mathrm{i}}(t), i=1, \ldots, L$, of duration $T$. Each $p_{\mathrm{i}}(t)$ includes one 'chip' of unit amplitude and duration $L / T$, in addition to $L-1$ chips of zero amplitude. A sequence of $p_{\mathrm{i}}(t)$ forms the chip waveform $b(t)$, which is scaled by the peak optical power $L P_{\mathrm{E}}$ and transmitted over the channel $h(t)$. The receiver employs a unit-energy filter $g(t)$, which is matched to $p_{1}(t)$ and then samples the filtered signal at rate $L / T$. The receiver 


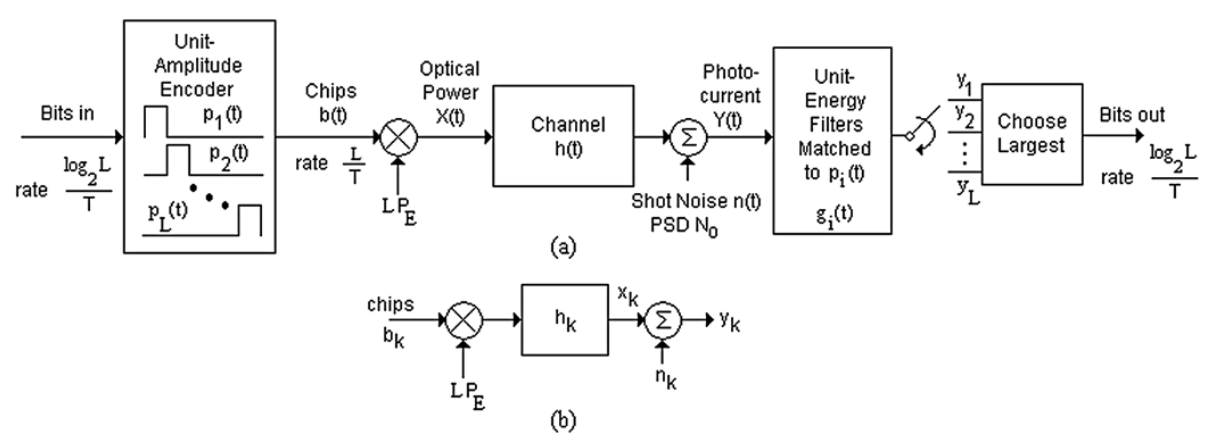

Figure 8 Block diagram of an L-PPM system. (a) Continuous-time block diagram of system. (b) Equivalent discrete-time block diagram of system sampled at the chip rate $L / T$.

chooses the largest among each group of the $L$ samples to obtain the detected symbol, which yields $\log _{2} L$ bits.

The system shown in Figure 8a can be simplified by converting to an equivalent discrete-time model sampled at the chip rate, as shown Figure $8 \mathrm{~b}[21]$. The combining of transmitter filter, multipath channel and receiver filter is described by the discrete-time impulse response $h_{k}$, which is given by

$$
h_{k}=\left.p(t) \otimes h(t) \otimes g(t)\right|_{t=k T / L},
$$

where $h(t)$ represents the combined impulse response when a conventional angle diversity receiver is employed. In this article, in order to obtain the output of the conventional angle diversity receiver (the combined channel response), two combining methods were considered: equal-gain combining (EGC) and selection combining (SC). While in EGC, $h(t)$ is obtained by summing the impulse response of each receiver branch; in $\mathrm{SC}, h(t)$ is the impulse response of the receiver branch with the lowest delay spread.

The receiver makes decisions based on the samples of the form:

$$
y_{k}=x_{k}+n_{k},
$$

where the signal samples $x_{k}$ are derived from the chip sequence $b_{k} \in\{0,1\}$ by

$$
x_{k}=L P_{E} b_{k} \otimes h_{k} .
$$

The convolution between $b_{k}$ and $h_{k}$ causes each nonzero transmitted chip to interfere with the samples within the same PPM symbol (intrasymbol interference) and also within adjoining PPM symbols (intersymbol interference). We will refer to these effects collectively as ISI. Since the unit-energy receiver filter $g(t)$ has a duration limited to $T / L$, the noise samples $n_{k}$ are independent, identically distributed Gaussian random variables with zero mean and variance $N_{0}=2 \sigma^{2}$. In [21], an expression for computing the bit error probability $\left(P_{b}\right)$ of an L-PPM link on an ISI channel was reported, which is expressed in terms of the well-known Q-function as

$$
P_{b}=\frac{L / 2}{L-1} P_{E}=\frac{L / 2}{L-1}\left[Q\left(\frac{x_{1}-x_{2}}{\sqrt{2 N_{0}}}\right)+Q\left(\frac{x_{1}-x_{3}}{\sqrt{2 N_{0}}}\right)+\ldots+Q\left(\frac{x_{1}-x_{L}}{\sqrt{2 N_{0}}}\right)\right]
$$

where $P_{\mathrm{E}}$ represents the symbol error probability. Particularising to $L=2$, the equation can be simplified as

$$
P_{b}=P_{E}=Q\left(\frac{x_{1}-x_{2}}{\sqrt{2 N_{0}}}\right)
$$

In order to obtain the average error probability for random input data, we average the $P_{b}$ given in (30) over all possible chip sequences $b_{k}$ having a duration equal to the length of the impulse response $h_{k}$. In the study, the effect of the symbols or bits farther than $\pm 6 T$ can be neglected [20], as was corroborated by the simulated impulse responses.

All studied receiver arrangements have a branch oriented vertically towards the ceiling and provide axial symmetry, i.e. the remaining branches are uniformly spaced in azimuth forming a $56^{\circ}$ angle with respect to the vertical branch. The receiver arrangements are described in Table 4. Each receiver branch is composed of a photodetector that employs a $\mathrm{CPC}$ with a $50^{\circ} \mathrm{FOV}$. The emitter was located in the centre of the room, $1 \mathrm{~m}$ above the floor and aimed vertically towards the ceiling, and 400 different locations for each receiver were simulated inside room B (see Figure 3). In the room, the ambient light is provided by four windows and six tungsten lamps in the ceiling, which are spaced $3 \mathrm{~m}$ apart. To calculate the incident optical power due to ambient light, each window is divided into small elements of area equal to $0.04 \mathrm{~m}^{2}$, and each element is modelled as a first-order Lambertian source. The lamps are modelled as Lambertian sources of second order. Table 1 shows the remaining parameters used for the simulations. 


\begin{tabular}{|c|c|c|}
\hline $\begin{array}{l}\text { Receiver } \\
\text { number }\end{array}$ & $\begin{array}{l}\text { Number of } \\
\text { branches }\end{array}$ & Structure description \\
\hline 1 & 1 & $\begin{array}{l}\text { One branch oriented vertically towards } \\
\text { the ceiling }\end{array}$ \\
\hline 3 & 3 & $\begin{array}{l}\text { One branch oriented vertically with two } \\
\text { uniformly spaced in azimuth }\end{array}$ \\
\hline 4 & 4 & $\begin{array}{l}\text { One branch oriented vertically with three } \\
\text { uniformly spaced in azimuth }\end{array}$ \\
\hline 5 & 5 & $\begin{array}{l}\text { One branch oriented vertically with four } \\
\text { uniformly spaced in azimuth }\end{array}$ \\
\hline 6 & 6 & $\begin{array}{l}\text { One branch oriented vertically with five } \\
\text { uniformly spaced in azimuth }\end{array}$ \\
\hline 7 & 7 & $\begin{array}{l}\text { One branch oriented vertically with six } \\
\text { uniformly spaced in azimuth }\end{array}$ \\
\hline 8 & 8 & $\begin{array}{l}\text { One branch oriented vertically with seven } \\
\text { uniformly spaced in azimuth }\end{array}$ \\
\hline 9 & 9 & $\begin{array}{l}\text { One branch oriented vertically with eight } \\
\text { uniformly spaced in azimuth }\end{array}$ \\
\hline
\end{tabular}

Figures 9 and 10 show the outage probability and the average error probability, respectively, for the eight conventional receiver arrangements for the 2-PPM links employing the EGC and SC methods. A bit rate of 100 Mbps was considered. When EGC is employed, the results reveal that starting with receiver number three, both parameters decrease as the number of receiver branches increases. Also, the results show that increasing the diversity order of the receiver above seven branches does not yield a significant improvement in terms of average error probability and outage probability, which tends toward a stable value of about $3 \%$, i.e. the conventional angle diversity receiver must consist of seven or more branches. In summary, the number of receiver branches selected is seven because the maximum number of detectors or branches in azimuth featuring a $\mathrm{CPC}$ with a $50^{\circ} \mathrm{FOV}$ that can be physically arranged is

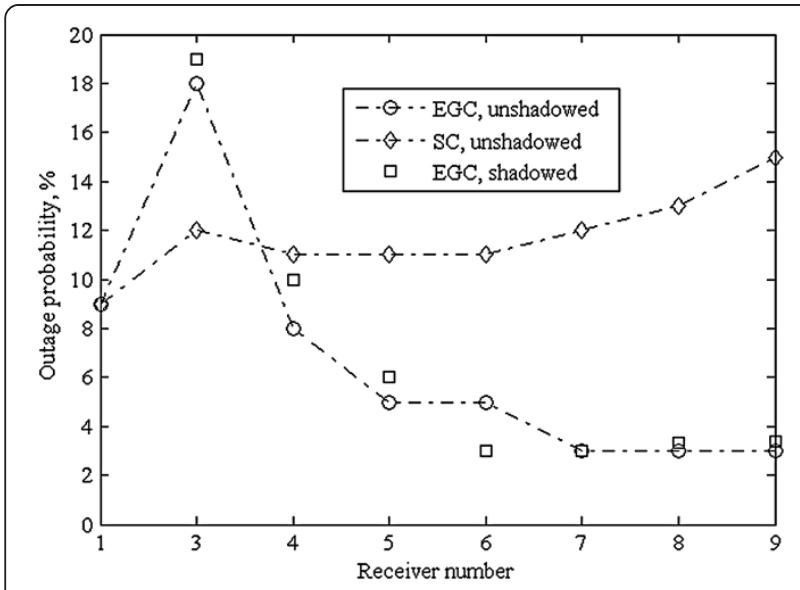

Figure 9 Outage probability for 2-PPM systems at 100 Mbps.

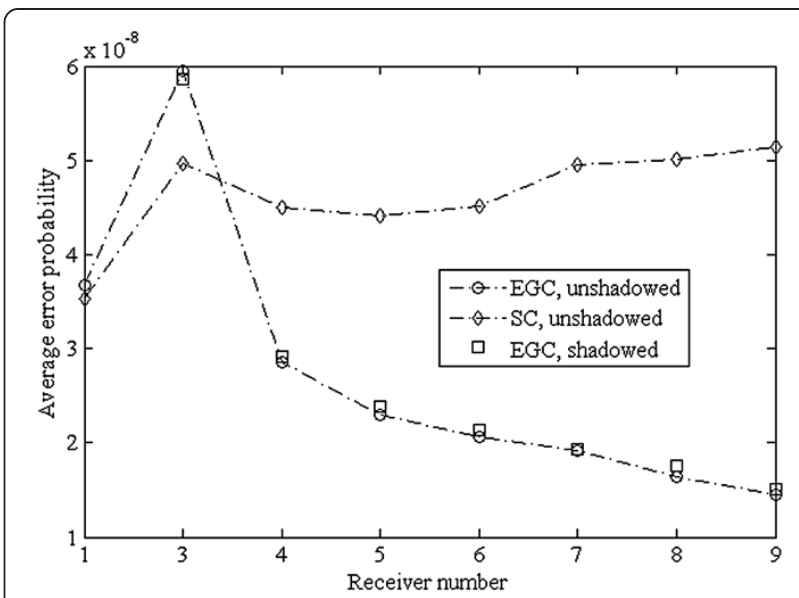

Figure 10 Average error probability evaluated at over 400 different locations.

six and because the increased number of branches does not yield a significant improvement in performance to justify the ensuing increase in the receiver's complexity. In fact, based on the results obtained in [20], there will be a number of branches greater than nine, above which the receiver performance degrades. When SC is used to combine the output of the angle diversity receivers, the variation in either parameter is not noticeable as a function of the receiver employed, i.e. the results yield no information to establish the diversity order of the receiver. Furthermore, SC is much more complex to implement than EGC. So, as to anticipate the effect resulting from the presence of obstacles in the conventional receiver arrangements considered, Figures 9 and 10 also show the three parameters when one of the receiver branches is obstructed. In this case, the plots were obtained employing EGC. As we can see, in every arrangement considered, the SNR dropped approximately $1 \mathrm{~dB}$, though the outage probability only decreased when receivers with fewer than seven detector elements were considered. For the remaining arrangements, both the outage probability and the average error probability will remain approximately equal to the values obtained without obstructing one of the branches.

The average error probability and the outage probability depend strongly on the emitted optical power $P_{\mathrm{E}}$ and the bit rate $R_{\mathrm{b}}$, which must be properly selected in order to achieve a desired error probability. Thus, an increase or a decrease in $P_{\mathrm{E}}$ and $R_{\mathrm{b}}$, respectively, will result in values smaller for both probabilities. As discussed in Section 2, the characteristics of an IR channel can be fully characterised by its impulse response, in which the channel gain (inverse of path loss) and rms delay spread can be directly extracted. In this case, the study was based on diffuse links with the emitter located at the centre of room B and oriented vertically upward. This is 
because of the fact that in this configuration, the path loss and rms delay spread are high and more sensitive to emitter-receiver distance changes [9]. In terms of SNR, Equation (30), which defines the bit error probability, can be rewritten as

$$
\begin{aligned}
P b & =P_{E}=Q\left(\sqrt{\frac{R^{2} P_{S}^{2}}{2 N_{0}}}\left(\frac{x_{1}-x_{2}}{R P_{S}}\right)\right) \\
& =Q\left(\frac{\sqrt{S N R}}{2}\left(x_{1}-x_{2}\right)\right) \\
& =Q\left[\sqrt{S N R}\left(\frac{1}{2}-d_{I S I}\right)\right],
\end{aligned}
$$

where $d_{\mathrm{ISI}} \in[0,1 / 2]$ represents the ISI effect due to $R_{\mathrm{b}}$, with $d_{\text {ISI }}$ being equal to 0 in the absence of ISI. Figures 11 and 12 show the outage probability and the average error probability evaluated at different bit rates and emitted optical powers, respectively. Both parameters were obtained for the 2-PPM links employing the EGC method. For a power of $0.4 \mathrm{~W}$, when the bit rate is increased, $d_{\mathrm{ISI}}$ is nearly $1 / 2$, providing an increase in the average error probability and therefore in the outage probability. When the bit rate is decreased, both probabilities are lower. On the other hand, we can observe that for a bit rate of $100 \mathrm{Mbps}$, the average error probability is improved when the power is increased. This follows from Equation (31), because an increase in $P_{\mathrm{E}}$ provides an increase in $P_{\mathrm{s}}$ and therefore in the SNR. In general, the results illustrate that the outage and error probabilities decrease when $P_{\mathrm{E}}$ and $R_{\mathrm{b}}$ are increased and decreased, respectively. Finally, all the plots shown in Figures 11 and 12 present a similar behaviour for all the bit rates and powers considered, i.e. regardless of $P_{\mathrm{E}}$ and $R_{\mathrm{b}}$, the same conclusions for the design of a conventional angle diversity receiver will be obtained.

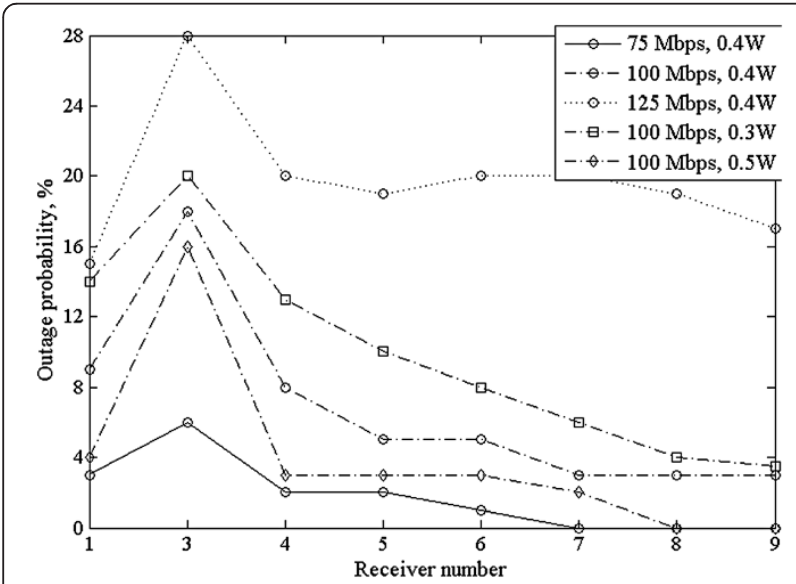

Figure 11 Outage probability evaluated at 75, 100 and 125 Mbps with $P_{\mathrm{E}}=\mathbf{0 . 4} \mathrm{W}$. For $R_{\mathrm{b}}=100 \mathrm{Mbps}$, power values of 0.3 and $0.5 \mathrm{~W}$ were also studied.

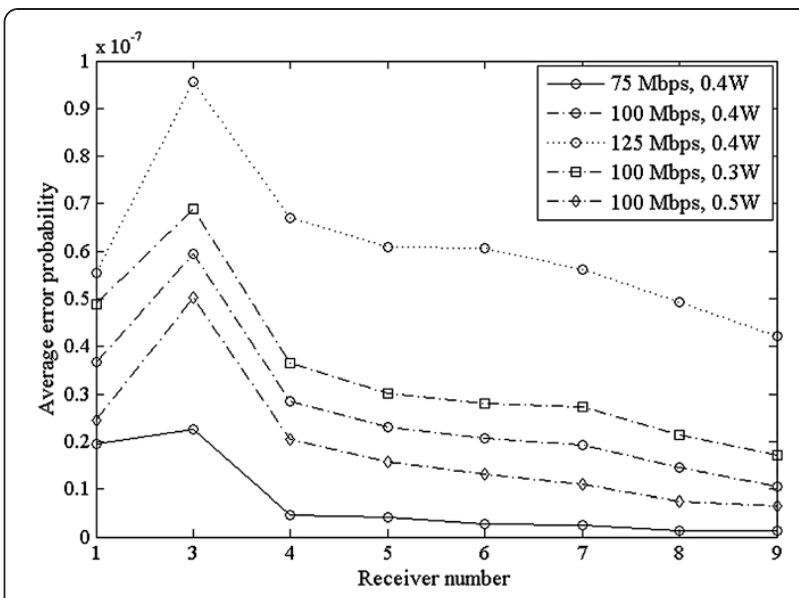

Figure 12 Average error probability evaluated at 75, 100 and $125 \mathrm{Mbps}$ with $\boldsymbol{P}_{\mathrm{E}}=\mathbf{0 . 4} \mathbf{W}$. For $R_{\mathrm{b}}=100 \mathrm{Mbps}$, power values of 0.3 and $0.5 \mathrm{~W}$ were also studied.

In short, according to the obtained results and assuming that a conventional receiver should have a detector oriented towards the ceiling, which is the main reflector of power, and that it must have axial symmetry, a receiver using angle diversity that relies on the use of concentrators with a $50^{\circ}$ FOV must consist of seven branches or detectors, one oriented vertically towards the ceiling, with the other six uniformly spaced in azimuth, forming a $56^{\circ}$ angle with respect to the vertical element (see Figure 1).

\section{Conclusions}

The propagation characteristics of an indoor infrared channel are fully described by the channel's impulse response, which depends on multiple factors such as the room geometry, the reflection pattern of surfaces, the emitter and receiver characteristics, and their relative locations. In this article, we studied those indoor IR links that are characterised by the use of conventional angle diversity receivers. A conventional receiver uses multiple photodetectors that are oriented in various directions, where each receiving element usually employs a filter and nonimaging concentrator, such as a CPC or hemispheric lens. Simulating an indoor IR channel can significantly benefit the design of high performance systems but requires computationally efficient algorithms and models that accurately match the characteristics of the channel elements. In this work, a Monte Carlo ray-tracing algorithm was used, which exhibits a high computational efficiency with respect to the previous algorithms, especially when a high temporal resolution and a large number of reflections are considered. This discussion can be extended to the evaluation of the shot noise variance from ambient light, since the incident optical power that results from the windows and 
lamps is also computed using the Monte Carlo raytracing algorithm, that is, in contrast to previous works, in this study, we considered not only the noise due to the incident optical power that directly reaches the receiver but also the optical power from multiple reflections. In short, the channel's impulse response and noise variance were determined with a higher degree of accuracy than in the previous research, which affects the calculation of the performance, in terms of SNR and bit error probability, of those IR communication systems that are characterised by the utilisation of conventional angle diversity receivers. In fact, obtaining an impulse response with a high temporal resolution is necessary in order to study systems operating at a high bit rate.

As discussed previously, in addition to the simulation algorithm, the models that accurately fit the characteristics of the channel elements are required. To this end, the models for the reflection pattern of surfaces and the effective signal collection area of a conventional angle diversity receiver were proposed, which more closely approximate real behaviour than those previously reported. The inclusion of these models was possible because of the high computational efficiency of the Monte Carlo ray-tracing algorithm. In conclusion, based on the results of the study of the influence of the conventional receiver parameters on the main IR channel parameters and on the effect that the increasing diversity order of the receiver (number of branches) has on the average error probability and the outage probability, as defined by the percentage of locations that correspond to an error probability greater than $10^{-9}$ employing 2-PPM systems with EGC and SC, a receiver structure composed of seven branches or detector elements was proposed, with one of the branches oriented towards the ceiling, and six at a $56^{\circ}$ elevation with a $60^{\circ}$ separation in azimuth. For each detector, a CPC with a $50^{\circ} \mathrm{FOV}$ must be used.

\section{Competing interests}

The authors declare that they have no competing interests.

\section{Acknowledgements}

This work was funded in part by the Spanish Ministerio de Ciencia e Innovación (project TEC2009-14059-C03-02/01/03), Plan E (Spanish Economy and Employment Stimulation Plan) and the Government of the Canary Islands (ProlD20100117).

\footnotetext{
Author details

${ }^{1}$ Departamento de Física Fundamental y Experimental, Electrónica y Sistemas, Universidad de La Laguna, La Laguna, Tenerife 38203, Spain. ${ }^{2}$ Instituto para el Desarrollo Tecnológico y la Innovación en Comunicaciones (IDeTIC), Universidad de Las Palmas de Gran Canaria, Las Palmas de Gran Canaria 35017, Spain
}

Received: 28 January 2013 Accepted: 20 August 2013

Published: 4 September 2013

\section{References}

1. JM Kahn, JR Barry, Wireless infrared communications. Proc. IEEE 85(2), 367-379 (1997). doi:10.1109/5.554222

2. FE Alsaadi, JMH Elmirghani, Adaptive mobile line strip multibeam MC-CDMA optical wireless system employing imaging detection in a real indoor environment. IEEE J. Sel. Areas Commun. 27(9), 1663-1675 (2009). doi:10.1109/JSAC.2009.091216

3. FE Alsaadi, JMH Elmirghani, Adaptive mobile multicarrier code division multiple access optical wireless systems employing a beam clustering method and diversity detection. IET Optoelectron. 4(3), 95-112 (2010). doi:10.1049/IET-OPT.2009.0006

4. AG Al-Ghamdi, JMH Elmirghani, Analysis of diffuse optical wireless channels employing spot-diffusing techniques, diversity receivers, and combining schemes. IEEE Trans. Commun. 52(10), 1622-1631 (2004). doi:10.1109/ TCOMM.2004.836509

5. JB Carruthers, JM Kahn, Angle diversity for nondirected wireless infrared communication. IEEE Trans. Commun. 48(6), 906-969 (2000). doi:10.1109/ 26.848557

6. S Jivkova, BA Hristov, M Kaverhrad, Power-efficient multispot-diffuse multiple-input-multiple-output approach to broad-band optical wireless communications. IEEE Trans. Veh. Technol. 53(3), 882-888 (2004). doi:10.1109/TVT.2004.825779

7. P Djahani, JM Kahn, Analysis of infrared wireless links employing multibeam transmitters and imaging diversity receivers. IEEE Trans. Commun. 48(12), 2077-2088 (2000). doi:10.1109/26.891218

8. CRAT Lomba, RT Valadas, AM De Oliveira Duarte, Sectored receivers to combat the multipath dispersion of the indoor optical channel, in Proceeding of Sixth IEEE International Symposium on Personal, Indoor and Mobile Communications (PIMRC '95), ed. by IEEE (IEEE, New York, 1995), pp. pp. 321-325

9. BR Mendoza, S Rodríguez, R Pérez-Jiménez, O González, E Poves, Considerations on the design of sectored receivers for wireless optical channels using a Monte-Carlo-based ray-tracing algorithm. IET Optoelectron. 1(5), 226-232 (2007). doi:10.1049/IET-OPT:20060062

10. JR Barry, JM Kahn, EA Lee, DG Messerschmitt, Simulation of multipath impulse response for indoor wireless optical channels. IEEE J. Sel. Areas Commun. 11(3), 367-379 (1993). doi:10.1109/49.219552

11. FJ López-Hernández, MJ Betancor, DUSTIN: a novel algorithm for the calculation of the impulse response on IR wireless indoor channels. Electron. Lett. 33(21), 1804-1805 (1997). doi:10.1049/EL:19971224

12. FJ López-Hernandez, R Pérez-Jiménez, A Santamaría, Ray-tracing algorithm for fast calculation of the channel impulse response on diffuse IR-wireless indoor channels. Opt Eng 39(10), 2775-2780 (2000). doi:10.1117/1.1287397

13. S Rodríguez, R Pérez-Jiménez, FJ López-Hernádez, O González, A Ayala, Reflection model for calculation of the impulse response on IR-wireless indoor channels using ray-tracing algorithm. Microw. Opt. Techn. Lett. 32(4), 296-300 (2002). doi:10.1002/MOP.10159

14. S Rodríguez, R Pérez Jiménez, O González, J Rabadán, BR Mendoza, Concentrator and lens models for calculating the impulse response on IRwireless indoor channels using a ray-tracing algorithmi. Microw. Opt. Techn. Lett 36(4), 262-267 (2003). doi:10.1002/MOP.10738

15. JR Barry, JM Kahn, Link design for nondirected wireless infrared communications. Appl. Opt. 34(19), 3764-3776 (1995). doi:10.1364/ AO.34.003764

16. O González, S Rodríguez, R Pérez-Jiménez, BR Mendoza, A Ayala, Error analysis of the simulated impulse response on indoor wireless optical channels using a Monte Carlo-based ray-tracing algorithm. IEEE Trans. Commun. 53, 124-130 (2005). doi:10.1109/TCOMM.2004.840625

17. O González, C Militello, S Rodríguez, R Pérez-Jiménez, A Ayala, Error estimation of the impulse response on diffuse wireless infrared indoor channels using a Monte Carlo ray-tracing algorithm. IEEE Proc. Optoelectron. 149(5/6), 222-227 (2002). doi:10.1049/IP-OPT:20020545

18. ST Jivkova, M Kavehrad, Multispot diffusing configuration for wireless infrared access. IEEE Trans. Commun. 48(6), 970-978 (2000). doi:10.1109/26.848558

19. MR Pakravan, M Kaverhad, Design considerations for broadband indoor infrared wireless communication systems. Int. J. Wirel. Inform. Netw. 2(4), 223-237 (1995). doi:10.1007/BF01538147 
20. YA Alqudah, M Kavehrad, Optimum order of angle diversity with equal-gain combining receivers for broad-band indoor optical wireless

communications. IEEE Trans. Veh. Technol. 53(1), 94-105 (2004). doi:10.1109/ TVT.2003.822023

21. MD Audeh, JM Kahn, JR Barry, Performance of pulse-position modulation on measured non-directed indoor infrared channels. IEEE Trans. Commun. 44(6), 654-659 (1996). doi:10.1109/26.506380

doi:10.1186/1687-1499-2013-221

Cite this article as: Rodríguez et al:: Design considerations of

conventional angle diversity receivers for indoor optical wireless

communications. EURASIP Journal on Wireless Communications and

Networking 2013 2013:221.

Submit your manuscript to a SpringerOpen ${ }^{\odot}$ journal and benefit from:

- Convenient online submission

- Rigorous peer review

- Immediate publication on acceptance

- Open access: articles freely available online

- High visibility within the field

- Retaining the copyright to your article

Submit your next manuscript at $\gg$ springeropen.com 